\title{
COMPARATIVE FACTORY ANALYSIS OF STANDARD FOUP CAPACITIES
}

\author{
Kranthi Mitra Adusumilli \\ Department of Operations Research \\ and Industrial Engineering \\ University of Texas \\ Austin, TX 78712, U.S.A.
}

\author{
Robert L. Wright \\ International SEMATECH Manufacturing Initiative \\ 2706 Montopolis Drive \\ Austin, TX 78741, U.S.A.
}

\begin{abstract}
Wafers in a $300-\mathrm{mm}$ semiconductor fabrication facility are transported throughout the factory in carriers called front opening unified pods (FOUPs). Two standard capacities of FOUPs are 25 and 13 wafers. This paper describes a simulation study designed to compare the performance of a factory employing different FOUP capacities. The main performance measure considered is work-in-process (WIP) and the resulting cycle time. Batching policy, order arrival rate, average order size, the Automated Material Handling System (AMHS) and the number of batch tools largely effect the performance of the models. Most of the empirical results show that the 25-wafer FOUP capacity provides a lower WIP level in a moderately loaded semiconductor factory.
\end{abstract}

\section{INTRODUCTION}

In a semiconductor fabrication facility, methods of improving factory efficiency are continuously explored. Such methods include increasing the utilization of resources, scheduling of lots, and determining the optimal batch size to improve the efficiency of a fab, while proceeding through the never-ending process of reducing cycle time. Numerous studies have been made to this end in the direction of finding the "optimal" lot and batch size. The standard lot sizes, under ideal conditions, are dictated by the capacity of the wafer carriers. Depending on the order size, the wafer carrier may not be completely filled to its capacity. Standard capacities of a FOUP are 25 and 13 wafers (I3001 Guidelines).

According to theory, a lot size of one is most advantageous for a system with sequential tools (Horn and Podgorski 1998). The amount of time a lot has to wait before it can be immediately processed at the tool decreases as the lot size decreases. This in turn reduces the total wait time for each lot, thereby decreasing cycle time.

As the size of the lot decreases, finer and more controlled batching is possible at batching tools, assuming the capacity of the tool in terms of lots is inversely related to the lot size. Therefore, control of the minimum batch size increases as the lot size decreases. This reduces the possibility of starvation of the tool and the time the downstream tool has to wait for a lot to arrive. These arguments indicate that a smaller lot size is beneficial.

However, it could be argued that as the lot size decreases and wafer starts remain constant, the amount of setups increase, lengthening cycle time. Smaller lot size implies more lots to be transferred and a higher number of lot movements to be handled. This increases the load on the AMHS. Waiting time of a lot at a tool is also a function of the queue length at that tool group. The various practical issues that include variation in order rates, processing times and tool down times, and the behavior of the AMHS make the validity of the above argument difficult to verify. Thus, an empirical analysis is performed through simulation study of fab models.

\section{DESCRIPTION}

\subsection{Modeling Software}

The software supplied by Brooks Automated Planning and Logistics for the discrete event simulation model described in this report is AutoSched Accelerated Processing ${ }^{\mathrm{TM}}$ $(A S A P)$ v 7.1 and AutoMod ${ }^{\mathrm{TM}}$ v9.1. ASAP $^{\mathrm{TM}}$ is an objectoriented modeling tool that uses a Windows-based Excel spreadsheet interface. The ASAP ${ }^{\mathrm{TM}}$ software describes the factory elements such as tools, stockers, products, processing logic (routes), and scheduling logic.

The AutoMod ${ }^{\mathrm{TM}}$ software incorporates real-time virtual graphic animation, helping to validate the model and communicate the design visually. The model communication module (MCM) provides the communication link (socket) between ASAP $^{\mathrm{TM}}$ and AutoMod ${ }^{\mathrm{TM}}$. The MCM keeps the two model components in continuous synchronization.

\subsection{Fab Layout}

The fabrication facility modeled is a generic $300 \mathrm{~mm}$ facility. The fab is completely automated using either overhead 
vehicles or conveyor transport. One main interbay with 24 intrabays are spaced evenly across the length of the fab. For further details about the basic model and the implicit assumptions, refer to SEMATECH technical reports (Campbell and Ammenheuser 2000) and (Quinn and Bass 1999).

Tools are grouped in a hybrid farm layout with the exception of metrology equipment. Tool dedication for lithography is modeled for the Active Area, Gate, Contact, Metal 1, and Via 1. To improve efficiency and reduce the constraint on the AMHS, metrology tools are distributed throughout the factory to decrease the amount of traveling required.

\subsection{Model Description and Assumptions}

The models used in this study are built on the models developed and enhanced at International SEMATECH. Three technologies $130 \mathrm{~nm}, 90 \mathrm{~nm}$ and $65 \mathrm{~nm}$ are modeled such to constitute $60 \%, 40 \%$ and $10 \%$ of the wafer starts respectively. Unless otherwise noted, base assumptions include an $18 \mathrm{k}$ wafer start per month factory, ten products per technology node, and a constant tool set. Each factory modeled uses a consistent size of lot carrier. In other words, 25-wafer FOUPs are not combined with 13-wafer FOUPs in the same factory. The model is based on a single technology processing fab with fewer operations and tools (Rust, Wright and Shopbell 2002).

Depending on the scenario, certain parameters are changed to maintain stability in the full factorial design of experiment. Changing parameters include the number of batch tools (Wet Benches and Furnaces), number of reticles and the batching policy. Other parameters that change based on a specific scenario are variations incorporated into the arrival rates of the orders, the number of products per technology, the order size of each product, and the AMHS. When a particular scenario is modeled, only one factor in the model is changed to attain a controlled experiment.

The modeling progression itself is a very iterative process. Individual models are manually synchronized so direct comparisons can be made. A particular change made in one model is extended to all models where comparisons are made.

Three key variables in the modeling work are significant to the results presented. Equipment batching strategies, order size, and the material handling system have the greatest impact on the models contained within the scope of this project.

Other than the Wet Bench and Furnace tool groups, all other tool groups are modeled as sequential tool families. The amount of processing time of a lot at a sequential tool is considered proportional to the lot size. At batch tools, the processing time for a batch is considered to remain constant regardless of the batch size.

Each tool can preload lot(s) for processing, while currently processing a lot. The capacity of the tool is fixed in the majority of the scenarios. However, as seen necessary for stability in some of the scenarios, the capacity of certain heavily loaded tool groups is increased in the models with 13-wafer FOUP carriers.

The order size is split in a "balanced" manner between various FOUPs. For example if the order size is 50 wafers, two FOUPs of 25 -wafer each are used, whereas with four FOUPs of 13-wafer capacity, two FOUPs with 12-wafers and 13-wafers each are used.

The simulation run length is between 120 and 150 days. Performance measures such as machine utilization and average cycle time are calculated only for the time frame across which the system is "stable". Transient time is not considered in the collection of statistics.

The random components incorporated in the model include equipment down time, variations in the processing times and inter arrival time of orders. In addition, the AMHS contributes to the already present randomness. Replications have not been considered. Long run-lengths and the low degree of randomness present in the model justify a lack of replications for this simulation exercise.

\section{THROUGH STOCKER}

\subsection{AMHS Logic}

The Through Stocker model demonstrates a first generation automated material handling system. Each intrabay contains space for two stockers. Depending on the loading of the intrabay, either one or both stockers are utilized.

As the lot carrier capacity decreases from 25 to 13 wafers, and order size increases, the number of lots increase potentially constraining the AMHS. After a lot is processed at a tool, the lot moves to a stocker in the current intrabay before it is moved to the stocker of the downstream tool group. Assuming one stocker is in use, the number of lot arrivals at a stocker is equal to twice the number of lots visiting the intrabay.

\subsection{Results and Discussion}

The 13-Wafer FOUP model has a greater tendency to be unstable (increasing WIP over time) compared to the 25Wafer FOUP fab. The WIP level for the 25-Wafer FOUP factory is comparatively low. As lot size decreases, the AMHS is considerably more loaded and the transportation time for lots and time spent waiting for transportation increases. This is confirmed by considering simulations of a factory with FOUP capacities of 20, 18 and 15 wafers.

The intrabay component of the AMHS is observed to cause an increase in cycle time for the following reasons. After a lot completes processing at a tool, the lot moves to the stocker. The lot waits to be task selected by a measurement or inspection tool ( $99 \%$ of the time) in the same bay. After being "task selected", the lot at the stocker will experience a delay due to heavy loading at the stocker and unavailable robotic resources before being transported to a 
downstream tool. Although simulations are completed to resolve the transportation issues, increasing the number of vehicles and increasing the number of load ports beyond a certain level has no positive effect in resolving the transportation related delays.

The load on the AMHS is decreased by considering all metrology steps to be integrated with the previous steps. As a result, the 13-wafer FOUP model performs favorably with an average WIP of 13,560 compared to an average WIP of 14,550 wafers with a FOUP capacity of 25 wafers. The above results indicate that when metrology tools are not considered or when integrated with batch tools, the load on the AMHS and particularly the stockers decreases.

Although the 13-wafer FOUP is more favorable in this one instance, no other modeling results indicated any advantage using the Through Stocker material handling system. Therefore, simulations using the Through Stocker AMHS ceased at this point.

\section{BATCHING}

\subsection{Relevance}

The above observations indicate that batching policies are important. Batching policies are controlled and balanced by the combination of the minimum batch size and maximum waiting time to form the batch.

The loading of batch tools and it's effect on other tool groups depend on the batching policy. The downstream tool is more susceptible to starve as the minimum batch size increases. However, the utilization on the tool group increases as the average batch size decreases. The effect of finer batching is prominent at lower loading of batch tool groups. It is necessary to use an effective batching policy to notice the advantages of FOUP capacity.

\subsection{Batch Tools}

The maximum batch size of a Furnace is modeled to be 4 lots and 8 lots for 25-Wafer and 13-Wafer FOUP carriers respectively. The total processing time of a batch is approximately $3 \mathrm{hrs}$. This includes a cooling time of a half hour.

A wet bench is modeled to hold up to 4 batches at a time. The maximum batch size is 2 lots and 4 lots for 25 Wafer and 13-Wafer FOUPs, respectively. Batching is in accordance with 'same setup' rules so that a setup is the same for all products of the same technology at a particular batch operation.

\section{TOOL-TO-TOOL AND CONVEYOR AMHS}

\subsection{Advantages}

As previously observed, the operation and use of a stocker is the main problem in the 'Through Stocker' AMHS. In the 'Tool-to-Tool' and 'Conveyor' AMHS models, the use of stockers is minimized. A lot is transported to a stocker only when the capacity of all the tools of the downstream tool group is exhausted, reducing the load on the AMHS. Advantages of 13-Wafer FOUPs can be analyzed under these third generation material handling systems where lots are delivered directly to available downstream equipment.

The advantage of the Tool-to-Tool AMHS is evident due to the decreased difference in WIP levels for 13-wafer and 25-wafer carriers when comparing Tool-to-Tool to Through Stocker. WIP levels under these AMHS scenarios are provided in Table 1. The batching policy is the same for all the products and has not been optimized in any sense.

\begin{tabular}{lcl}
\multicolumn{3}{c}{ Table 1: WIP for Different AMHS Scenarios } \\
\hline AMHS & FOUP Capacity & WIP(/wafers) \\
\hline Tool-to-Tool & 13 & 13,260 \\
Tool-to-Tool & 25 & 12,000 \\
Through Stocker & 13 & 16,900 \\
Through Stocker & 25 & 15,000 \\
\hline
\end{tabular}

\subsection{Order Size}

The number of lots in the model is dependent on the average order size and the total expected wafer starts per month. The percentage of the order size referenced in Table 2 and factoring in the total wafer starts determines the load on tools, more significantly on batch equipment. The number of lots is proportional to the following expression

$$
\sum(N) *(\% \text { of Order size) /(Order Size) }
$$

where $N$ is the number of lots resulting from splitting an incoming order. As the FOUP capacity decreases, the load increases in accordance with the above expression. For a small incoming order, the load on batch tools is greater in the 25-Wafer FOUP case as the average batch size has a proportionately lower number of wafers compared to the 13-Wafer case.

\subsubsection{Results and Observation}

Two different scenarios, 13-wafer and 25-wafer FOUPs have been considered using a simple batching policy applied to all the products in each of the models. Table 2 summarizes the results. In each of the scenarios, seven products of each technology are modeled. For this comparison and analyses, the Tool-to-Tool automated material handling system is used.

Irrespective of the order size and order configuration, and using the simple batching policy, the 25-Wafer FOUP is most beneficial. However, as the average order size increases in Scenario two, the WIP gap between the 13-wafer and 25-wafer models narrows. Conclusions cannot be drawn from this fact unless the batching policy is analyzed carefully. 
Table 2: Two Scenarios of Varying Order Size

\begin{tabular}{|c|c|c|c|c|c|c|c|c|}
\hline FOUPs capac- & \multicolumn{4}{|c|}{13 Wafers } & \multicolumn{4}{|c|}{25 Wafers } \\
\hline Order Size & 50 & 25 & 20 & 15 & 13 & 9 & 6 & 2 \\
\hline \multicolumn{9}{|l|}{ Scenario 1} \\
\hline $\begin{array}{c}\% \text { of wafer } \\
\text { starts }\end{array}$ & 0 & 20 & 7.5 & 7.5 & 30 & 20 & 10 & 5 \\
\hline WIP & \multicolumn{4}{|c|}{11,870} & \multicolumn{4}{|c|}{8,768} \\
\hline \multicolumn{9}{|l|}{ Scenario 2} \\
\hline $\begin{array}{c}\begin{array}{c}\% \text { of wafer } \\
\text { starts }\end{array} \\
\end{array}$ & 10 & 15 & 25 & 15 & 20 & 10 & 0 & 5 \\
\hline WIP & \multicolumn{5}{|c|}{12,010} & \multicolumn{3}{|c|}{9,660} \\
\hline
\end{tabular}

\subsection{Batching and Results}

For batching simulations, both Tool-to-Tool and Conveyor automated material handling systems are considered. Various scenarios are analyzed to consider the effect of batching on the fab. These scenarios are hypothetical and are generated by a change in the batching policy. Analyses of these scenarios is necessary to assess the effect of factors on the ideal FOUP capacity. Unless otherwise mentioned, the parameters of the batching policy are proportioned to FOUP capacity to the extent possible.

\subsubsection{Removal of Batch Tools}

In this scenario, all of the batching processing steps and operations are eliminated. As a result, WIP levels are equivalent regardless of the AMHS and the FOUP capacity. The corresponding WIP levels are not affected by either the considerable increase in setup time required for tools or the heavy loading of the AMHS as the number of lots approximately double. The percentage of time that a lot spends waiting for transportation or in transportation is significantly lowered.

\subsubsection{Single Batch Size}

The minimum batch size is considered equivalent to the maximum batch size. That is, the batch size is forced to the maximum batch size allowed by a batch tool. This scenario is the most stable in the sense that it requires the minimum number of batching tools. This policy was applied across all technologies and products. As a result, a technology with lower order rates requires more time to form a batch. In this scenario, the 25-Wafer FOUP is more beneficial no matter which AMHS is employed.

\subsubsection{Varying Batch Size}

The minimum batch size range varies from one lot to one lot less than the capacity of the batch tool. Also, the maximum waiting time is varied. For flexibility, more batch tools are added in the model. Various combinations of the batch size and waiting time are modeled to decrease WIP levels. The WIP levels vary considerably in the 13-wafer FOUP model when a change is made to parameters.

Under a certain batching policy, the 13-Wafer FOUP and 25-Wafer FOUP models have almost the same WIP levels, although the idle percentage of batch tools is lower in the 25-Wafer FOUP case. This indicates that under an "optimal" batching policy, comparisons of the two different fab models cannot be predicted.

In the context of this work, we consider a good batching policy to be a policy in which utilization is maximized in batch tools while maintaining level WIP.

\section{AGGREGATE EXPERIMENTS}

Two specific scenarios with "good" batching policies and a mix of order sizes are modeled. In these scenarios, the number of wafer starts per month is 18,000 wafers with 30 products per technology. The Conveyor AMHS is considered for both scenarios. In the first scenario, the order size is as provided in Table 3. As observed, under the right conditions and batching policy, the 13-wafer FOUP indicates a slight decrease in the number of wafers in WIP compared to the 25-wafer FOUP.

Table 3: 13-Wafer FOUP Advantages

\begin{tabular}{|c|c|c|c|c|}
\hline \multicolumn{2}{|c|}{ WIP (FOUPs) } & \multicolumn{3}{|c|}{ Order Size } \\
\hline 13 & 25 & $130 \mathrm{~nm}$ & $90 \mathrm{~nm}$ & $65 \mathrm{~nm}$ \\
\hline 9,115 & 9,215 & 25 & 13 & 13 \\
\hline
\end{tabular}

In the second scenario, the batching policy was optimized, looking at various combinations of batching parameters. An order size of 25 wafers is constant for all products. Overall, the results are similar to Table 3 with only a slight decrease of $1-2 \%$ in WIP from the 25 -wafer FOUP to the 13-wafer FOUP. These two scenarios provide the only results where the 13-wafer FOUP is optimal.

As better batching policies were employed, it was observed a smaller number of batch tools are required to maintain stability. The number of batch tools drive the batch sizes through the batching policy. Hence, finer batching cannot be accomplished when the number of batch tools are at high or low extremes. For example, in the second scenario, when a large number of wet benches are modeled, good batching policies resulted in average batch size of one, regardless of the FOUP size; an advantage for the 13-wafer FOUP model.

In these scenarios, WIP levels are observed to decrease by as much as $20 \%$ in simulations where metrology tools were modeled as if they were integrated with other tools.

\section{CONCLUSIONS}

Tool-to-Tool and Conveyor AMHS are improved material handling systems to be considered for the fab modeled. 
Batch tools and metrology tools are the main tool groups adding considerable waiting time and further increasing cycle time. Batch tools have lengthy processing times and metrology tools require the AMHS to travel to a number of different bays. Based on the results from the Through Stocker model, it can be inferred that integrated metrology tools create ideal conditions for use of 13-Wafer FOUPs.

Another important factor to be considered is the average order size and the order size distribution. Depending on the order size distribution, batching policies can be applied differently to different products and technologies as employed in scenario one of section six. Presence of a minimal number of batch tools, negates the advantage gained due to smaller lot sizes.

With the "right" batching policy and under conditions of low utilization, the13-Wafer FOUP model might prove to be beneficial as can be found in certain cases. Batching policies proved to be the most significant factor in decreasing the WIP level. In general, it is found that 25-Wafer FOUPs are beneficial. Extensive modeling of the various material handling systems while varying parameters did not provide conclusive evidence that the 13-wafer FOUP is advantageous when the factory modeled contains all 13-wafer FOUPs. Even under the most ideal conditions modeled, the 13-wafer FOUP model performed only marginally better.

\section{ACKNOWLEDGMENTS}

The authors are grateful to acknowledge the numerous suggestions and insights from Senior Fellow, Walt Trybula, of International SEMATECHs Lithography division and Mike Schwartz of International SEMATECH Manufacturing Initiative.

\section{REFERENCES}

Campbell, E., and J. Ammenheuser. 2000. 300mm Factory Layout and Material Handling Modeling: Phase II Report. Technology Transfer Document, International SEMATECH. Available online via $<$ http: //www . sematech.org/docubase/document/ 3848beng.pdf $>$ [access-ed May 1, 2004].

Horn, G., and W. A. Podgorski. 1998. What Gain from Small Batch Manufacturing? Semiconductor Fabtech. $8^{\text {th }}$ ed. 35-37.

I300I Guidelines on $300 \mathrm{~mm}$ Process Tool Mechanical Interfaces for Wafer Lot Delivery, Buffering, and Loading. Technology Transfer Document, International $300 \mathrm{~mm}$ Initiative. Available online via $<$ http:// www. sematech.org/docubase/abstracts/ $3298 \mathrm{axfr}$.htm $>$ [accessed May 1, 2004]

Quinn, T., and E. Bass. 1999. 300mm Factory Layout and Material Handling Modeling: Phase I Report. Technology Transfer Document, International SEMATECH. Available online via $<$ http://www. sematech. org/docubase/document/3688beng . pdf> [accessed May1, 2004].

Rust, K., R. Wright, and M. Shopbell. 2002. Comparative Analysis of $300 \mathrm{~mm}$ Automated Handling Systems (AMHS). In Proceeding of the International Conference on Modeling and Analysis of Semiconductor Manufacturing, ed. G. T. Mackulak, J. W. Fowler, A. Schömig, 240-245.

\section{AUTHOR BIOGRAPHIES}

KRANTHI MITRA ADUSUMILLI is a Modeling and Simulation Analyst at International SEMATECH for Manufacturing Initiative. Employed by SEMATECH as an intern since January 2003, he has developed simulation models to closely represent futuristic fabs and conducted simulation studies to assess the effect under various conditions. He received his BTech. in Production and Industrial Engineering from the Indian Institute of Technology Delhi, India. Presently, he is pursuing his doctoral studies in Operations Research and Industrial Engineering at the University of Texas.

ROBERT WRIGHT manages the factory simulation program and cost modeling projects for the International SEMATECH Manufacturing Initiative of International SEMATECH. Robert has more than eight years of cost modeling and discrete event simulation experience and is a member of the technical staff. He received his BBA in Management and his MST in Industrial Technology from Texas State University. Robert teaches courses in Planning Advanced Technology Facilities and Engineering Economics at Texas State University as an adjunct faculty member. 